\section{Allergie gegen die Tage}

sraelische Gynäkologen führten bei 20

Frauen mit prämenstruellem Syndrom Intradermaltests mit Estradiolvalerat und Progesteron durch und konnten in allen 20 Fällen allergische Früh- und Spätphasenreaktionen beobachten. Bei einer Kontrollgruppe mit zehn Frauen ohne Beschwerden vor den Tagen verlief der Test negativ. Eine anschließende Desensibilisierung von 15 Patientinnen der ersten Gruppe gegen die Sexualhormone führte ausnahmslos zu einer Besserung der Symptome, insbesondere reduzierten sich auch prämenstruelle Hautreaktionen wie Pruritus vulvae, Hyperpigmentation oder Akne. Die Autoren vermuten, mit ihrer Entdeckung einem entscheidenden Pathomechanismus des

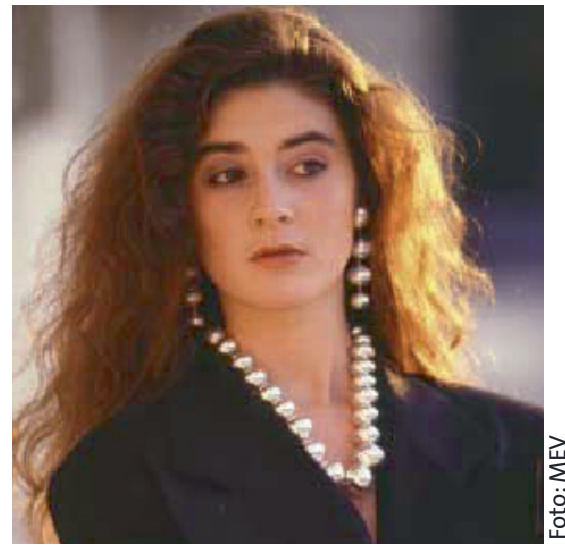

prämenstruellen Syndroms auf die Spur gekommen zu sein.

\section{es}

Itsekson A et al. J Reprod Med 2004; 49: 195-9

\title{
Risikofaktor erhöhtes Nabelschnur-IgE
}

- $\mathrm{t}$ orscher der Michigan State University bestimmten in den Jahren 1989 und 1990 bei mehr als 1.300 Geburten die IgE-Konzentration im Nabelschnurblut. Im Alter von 1 bis 2 sowie 4 und 10 Jahren wurden dann bei den Kindern Pricktests mit gängigen Aeroallergenen durchgeführt, das Vorliegen von Asthma wurde dokumentiert. Bei den 4- und den 10-jährigen Kindern korrelierte ein erhöhter Nabelschnur-IgE-Wert signifikant mit einer allergischen Sensibilisierung - ein
Ergebnis, das schon aus anderen Studien bekannt ist. Neu war dagegen, dass sich ein Zusammenhang zwischen Nabelschnur-IgE und Asthma zeigte, allerdings erst im Alter von 10 Jahren (Odds Ratio 1,66, 95\%-Konfidenzintervall 1,05-2,62). Die Korrelation war noch deutlicher, wenn bei den Asthmatikern keine allergische Sensibilisierung vorlag

Sadeghnejad A et al. Thorax 2004; 59 : 936-42

\section{Dicke Luft in der Eishalle}

- in Sportmediziner aus dem US-Bun- desstaat Pennsylvania dokumentierte über 4 Jahre hinweg die Lungenfunktionsparameter von 14 Profi-Eishockeyspielerinnen und neun Langläuferinnen. Während sich bei den nordischen Athletinnen im Mittel keine Änderung ergab, verschlechterten sich bei den Eishockeyspielerinnen die Werte kontinuierlich, insbesondere nahm der forcierte exspiratorische Fluss (FEF) ab. Sehr wahrscheinlich, so die Erklärung des Autors, werden die Lungen der Hockeyspielerinnen von den Abgasen der mit Verbrennungsmotoren ausgestatteten Eismaschinen, die während Training und Spiel die Eisfläche glätten, in Mitleidenschaft gezogen. Eine Analyse der Hallenluft ergab in der Tat hohe Anteile feiner und ultrafeiner Partikel.

Rundell KW. Inhal Toxicol 2004; 16: $117-23$

\section{Schaden am Auge}

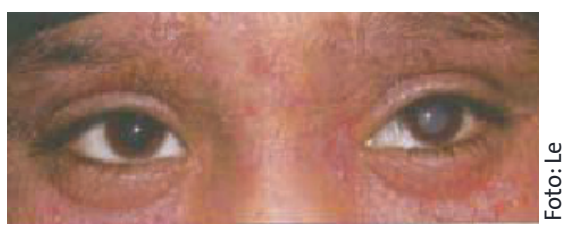

_ ine 25 jährige Frau stellt sich wegen - Visusverlust am linken Auge in der Klinik vor. Es fallen ein schweres nässendes Ekzem der Orbitalregionen und eine beidseitige Katarakt, links ausgeprägter als rechts, auf. Bei der Anamneseerhebung stellt sich heraus, dass sie über ein Jahr hinweg 1\%ige Hydrocortisonsalbe im Bereich der Lider angewandt hat. Die Erzeugung einer Katarakt durch systemisch oder lokal an der Konjunktiva applizierte Glukokortikoide ist wohl bekannt, weniger dagegen der Effekt von Glukokortikoid-Cremes und -Salben, die in der Orbitalregion an der Haut angewendet werden.

hsf 